\title{
A note on general solutions to a hyperbolic-cotangent class of systems of difference equations
}

\section{Stevo Stević1, $2^{*}$}

\section{"Correspondence: sstevic@ptt.rs 'Mathematical Institute of the Serbian Academy of Sciences, Knez Mihailova 36/III, 11000 Beograd, Serbia \\ ${ }^{2}$ Department of Medical Research, China Medical University Hospital, China Medical University, Taichung 40402, Taiwan, People's Republic of China}

\begin{abstract}
Recently there has been some interest in difference equations and systems whose forms resemble some trigonometric formulas. One of the classes of such systems is the so-called hyperbolic-cotangent class of systems of difference equations. The corresponding two-dimensional class has two delays denoted by $k$ and $I$. So far the class has been studied for the case $k \neq l$, and it was shown that it is practically solvable when $\max \{k, l\} \leq 2$. In this note we show practical solvability of the system in the case $k=l$, not only for small values of $k$ and $l$, but for all $k=l \in \mathbb{N}$, which is the first result of such generality.
\end{abstract}

MSC: 39A45

Keywords: System of difference equations; Solvable systems; Practical solvability

\section{Introduction}

Let, as usual, $\mathbb{N}$ denote the set of natural numbers, $\mathbb{N}_{0}$ of nonnegative integers, $\mathbb{Z}$ of integers, $\mathbb{R}$ of reals, $\mathbb{C}$ of complex numbers, and let $k=\overline{l, m}$, where $l, m \in \mathbb{Z}, l \leq m$, denote the set of all integers $k$ such that $l \leq k \leq m$.

Finding closed-form formulas for solutions to difference equations and systems is one of the first topics investigated in the branch of mathematics. One of the first solvable difference equations appearing in the literature was the following:

$$
a_{n}=c_{2} a_{n-1}+c_{1} a_{n-2}, \quad n \geq 2,
$$

where $c_{1}, c_{2} \in \mathbb{R}$,

$$
c_{1} \neq 0 \quad \text { and } \quad c_{2}^{2} \neq-4 c_{1} \text {. }
$$

In [1] de Moivre showed that, under the conditions in (2), a general solution to equation (1) is

$$
a_{n}=\frac{\left(a_{1}-s_{2} a_{0}\right) s_{1}^{n}+\left(s_{1} a_{0}-a_{1}\right) s_{2}^{n}}{s_{1}-s_{2}}, \quad n \in \mathbb{N}_{0},
$$

(c) The Author(s) 2020. This article is licensed under a Creative Commons Attribution 4.0 International License, which permits use, sharing, adaptation, distribution and reproduction in any medium or format, as long as you give appropriate credit to the original author(s) and the source, provide a link to the Creative Commons licence, and indicate if changes were made. The images or other third party material in this article are included in the article's Creative Commons licence, unless indicated otherwise in a credit line to the material. If material is not included in the article's Creative Commons licence and your intended use is not permitted by statutory regulation or exceeds the permitted use, you will need to obtain permission directly from the copyright holder. To view a copy of this licence, visit http://creativecommons.org/licenses/by/4.0/. 
where $s_{j}, j=1,2$, are the roots of the polynomial $Q(s)=s^{2}-c_{2} s-c_{1}$. From $c_{1} \neq 0$, we see that $Q(s)$ has two nonzero roots, whereas the second condition in (2) shows that they are distinct.

The method in [1] was used in [2] for finding general solutions to some other linear difference equations, including the solution to equation (1) in the case $c^{2}+4 c_{1}=0$. For more information on linear difference equations, consult for instance [3-8]. For some recent applications of linear difference equations in solvability of nonlinear ones, see for instance [9-19]. Sometimes it is not possible to find general solutions to difference equations and systems, but it is possible to find their invariants [20-25]. There are also cases when they can help in solvability [26, 27]. For various applications, see for instance [28-32].

There are classes of difference equations which are similar to some trigonometric formulas. The similarity suggests potential solvability of the difference equations in the form. An example of such a difference equation appeared long time ago in [33]. One of such classes is the hyperbolic-cotangent class of difference equations (see, for instance, [15]).

Motivated by the hyperbolic-cotangent class of equations, by our paper [11], and in general by papers by Papaschinopoulos and Schinas on systems of difference equations (see, e.g., [20-25, 34-37]), we have initiated an investigation of the corresponding classes of hyperbolic-cotangent systems of difference equations, that is, of the following ones:

$$
x_{n+1}=\frac{u_{n-k} v_{n-l}+a}{u_{n-k}+v_{n-l}}, \quad y_{n+1}=\frac{w_{n-k} s_{n-l}+a}{w_{n-k}+s_{n-l}}, \quad n \in \mathbb{N}_{0},
$$

where $k, l \in \mathbb{N}_{0}$, parameter $a$ and initial values are complex numbers, and where $u_{n}, v_{n}, w_{n}$, and $s_{n}$ are $x_{n}$ or $y_{n}$.

We have shown that for some values of $k$ and $l$, all the systems in (4) are solvable in closed form. More concretely, in [18] and [19] the systems when $k=0$ and $l=1$ were solved. Another solution to the solvability problem in this case was given in [13]. Further, in [12] the systems in the case when $k=1$ and $l=2$ were solved, and finally in [38] the systems were solved in the case $k=0$ and $l=2$. The methods used therein are closely related to those used in the study of product-type systems (see, for instance, [39] as well as the related references therein).

To complete a solution to the solvability problem for the systems in the case $\max \{k, l\} \leq$ 2 , it is needed to deal with the case when $k=l$. Hence, in this paper we study the following systems of difference equations:

$$
x_{n+1}=\frac{u_{n-k} v_{n-k}+a}{u_{n-k}+v_{n-k}}, \quad y_{n+1}=\frac{w_{n-k} s_{n-k}+a}{w_{n-k}+s_{n-k}}, \quad n \in \mathbb{N}_{0},
$$

where $k \in \mathbb{N}_{0}$.

Since for $a=0$ some simple and obvious changes of variables transform the systems in (5) to some homogeneous linear ones with constant coefficients, the case will be omitted (see also [13]).

First note that if $k \in \mathbb{N}$, then system (5) is with interlacing indices [14, 16]. Namely, let

$$
\begin{array}{lll}
x_{m}^{(i)}=x_{m(k+1)-i}, & y_{m}^{(i)}=y_{m(k+1)-i}, & u_{m}^{(i)}=u_{m(k+1)-i}, \\
v_{m}^{(i)}=v_{m(k+1)-i}, & w_{m}^{(i)}=w_{m(k+1)-i}, & s_{m}^{(i)}=s_{m(k+1)-i},
\end{array}
$$


for $m \in \mathbb{N}_{0}$, and $i=\overline{0, k}$. Then it is easy to see that

$$
x_{m+1}^{(i)}=\frac{u_{m}^{(i)} v_{m}^{(i)}+a}{u_{m}^{(i)}+v_{m}^{(i)}}, \quad y_{m+1}^{(i)}=\frac{w_{m}^{(i)} s_{m}^{(i)}+a}{w_{m}^{(i)}+s_{m}^{(i)}}
$$

for every $m \in \mathbb{N}_{0}$, and $i=\overline{0, k}$. Hence, the sequences $\left(x_{m}^{(i)}, y_{m}^{(i)}\right)_{m \in \mathbb{N}_{0}}, i=\overline{0, k}$, are solutions to system (5) with $k=0$, implying that each solution to (5) consists of $k+1$ unrelated solutions to the system in the case $k=0$. This shows that it is of some interest to study only the systems in the case when $k=0$, which is done in the sections that follow.

\section{Product-type systems associated with the ones in (5)}

The systems of difference equations in (5) with $k=0$ are naturally connected to some product-type ones. To show this, first note that the following simple relations hold:

$$
x_{n+1} \pm \sqrt{a}=\frac{\left(u_{n} \pm \sqrt{a}\right)\left(v_{n} \pm \sqrt{a}\right)}{u_{n}+v_{n}} \text { and } y_{n+1} \pm \sqrt{a}=\frac{\left(w_{n} \pm \sqrt{a}\right)\left(s_{n} \pm \sqrt{a}\right)}{w_{n}+s_{n}}
$$

for $n \in \mathbb{N}_{0}$, from which we obtain

$$
\frac{x_{n+1}+\sqrt{a}}{x_{n+1}-\sqrt{a}}=\frac{u_{n}+\sqrt{a}}{u_{n}-\sqrt{a}} \cdot \frac{v_{n}+\sqrt{a}}{v_{n}-\sqrt{a}}, \quad \frac{y_{n+1}+\sqrt{a}}{y_{n+1}-\sqrt{a}}=\frac{w_{n}+\sqrt{a}}{w_{n}-\sqrt{a}} \cdot \frac{s_{n}+\sqrt{a}}{s_{n}-\sqrt{a}}
$$

for $n \in \mathbb{N}_{0}$.

Now note that the system of difference equations (7) consists of the following nine ones:

$$
\begin{aligned}
& \frac{x_{n+1}+\sqrt{a}}{x_{n+1}-\sqrt{a}}=\left(\frac{x_{n}+\sqrt{a}}{x_{n}-\sqrt{a}}\right)^{2}, \quad \frac{y_{n+1}+\sqrt{a}}{y_{n+1}-\sqrt{a}}=\left(\frac{x_{n}+\sqrt{a}}{x_{n}-\sqrt{a}}\right)^{2}, \\
& \frac{x_{n+1}+\sqrt{a}}{x_{n+1}-\sqrt{a}}=\left(\frac{x_{n}+\sqrt{a}}{x_{n}-\sqrt{a}}\right)^{2}, \quad \frac{y_{n+1}+\sqrt{a}}{y_{n+1}-\sqrt{a}}=\frac{y_{n}+\sqrt{a}}{y_{n}-\sqrt{a}} \cdot \frac{x_{n}+\sqrt{a}}{x_{n}-\sqrt{a}}, \\
& \frac{x_{n+1}+\sqrt{a}}{x_{n+1}-\sqrt{a}}=\left(\frac{x_{n}+\sqrt{a}}{x_{n}-\sqrt{a}}\right)^{2}, \quad \frac{y_{n+1}+\sqrt{a}}{y_{n+1}-\sqrt{a}}=\left(\frac{y_{n}+\sqrt{a}}{y_{n}-\sqrt{a}}\right)^{2}, \\
& \frac{x_{n+1}+\sqrt{a}}{x_{n+1}-\sqrt{a}}=\frac{x_{n}+\sqrt{a}}{x_{n}-\sqrt{a}} \cdot \frac{y_{n}+\sqrt{a}}{y_{n}-\sqrt{a}}, \quad \frac{y_{n+1}+\sqrt{a}}{y_{n+1}-\sqrt{a}}=\left(\frac{x_{n}+\sqrt{a}}{x_{n}-\sqrt{a}}\right)^{2}, \\
& \frac{x_{n+1}+\sqrt{a}}{x_{n+1}-\sqrt{a}}=\frac{x_{n}+\sqrt{a}}{x_{n}-\sqrt{a}} \cdot \frac{y_{n}+\sqrt{a}}{y_{n}-\sqrt{a}}, \quad \frac{y_{n+1}+\sqrt{a}}{y_{n+1}-\sqrt{a}}=\frac{y_{n}+\sqrt{a}}{y_{n}-\sqrt{a}} \cdot \frac{x_{n}+\sqrt{a}}{x_{n}-\sqrt{a}}, \\
& \frac{x_{n+1}+\sqrt{a}}{x_{n+1}-\sqrt{a}}=\frac{x_{n}+\sqrt{a}}{x_{n}-\sqrt{a}} \cdot \frac{y_{n}+\sqrt{a}}{y_{n}-\sqrt{a}}, \quad \frac{y_{n+1}+\sqrt{a}}{y_{n+1}-\sqrt{a}}=\left(\frac{y_{n}+\sqrt{a}}{y_{n}-\sqrt{a}}\right)^{2}, \\
& \frac{x_{n+1}+\sqrt{a}}{x_{n+1}-\sqrt{a}}=\left(\frac{y_{n}+\sqrt{a}}{y_{n}-\sqrt{a}}\right)^{2}, \quad \frac{y_{n+1}+\sqrt{a}}{y_{n+1}-\sqrt{a}}=\left(\frac{x_{n}+\sqrt{a}}{x_{n}-\sqrt{a}}\right)^{2} \text {, } \\
& \frac{x_{n+1}+\sqrt{a}}{x_{n+1}-\sqrt{a}}=\left(\frac{y_{n}+\sqrt{a}}{y_{n}-\sqrt{a}}\right)^{2}, \quad \frac{y_{n+1}+\sqrt{a}}{y_{n+1}-\sqrt{a}}=\frac{y_{n}+\sqrt{a}}{y_{n}-\sqrt{a}} \cdot \frac{x_{n}+\sqrt{a}}{x_{n}-\sqrt{a}}, \\
& \frac{x_{n+1}+\sqrt{a}}{x_{n+1}-\sqrt{a}}=\left(\frac{y_{n}+\sqrt{a}}{y_{n}-\sqrt{a}}\right)^{2}, \quad \frac{y_{n+1}+\sqrt{a}}{y_{n+1}-\sqrt{a}}=\left(\frac{y_{n}+\sqrt{a}}{y_{n}-\sqrt{a}}\right)^{2}
\end{aligned}
$$

for $n \in \mathbb{N}_{0}$. 
The following substitutions

$$
\zeta_{n}=\frac{x_{n}+\sqrt{a}}{x_{n}-\sqrt{a}} \quad \text { and } \quad \eta_{n}=\frac{y_{n}+\sqrt{a}}{y_{n}-\sqrt{a}}
$$

yield

$$
x_{n}=\sqrt{a} \frac{\zeta_{n}+1}{\zeta_{n}-1} \text { and } y_{n}=\sqrt{a} \frac{\eta_{n}+1}{\eta_{n}-1},
$$

so that (8)-(16) become

$$
\begin{array}{ll}
\zeta_{n+1}=\zeta_{n}^{2}, & \eta_{n+1}=\zeta_{n}^{2}, \\
\zeta_{n+1}=\zeta_{n}^{2}, & \eta_{n+1}=\eta_{n} \zeta_{n}, \\
\zeta_{n+1}=\zeta_{n}^{2}, & \eta_{n+1}=\eta_{n}^{2}, \\
\zeta_{n+1}=\zeta_{n} \eta_{n}, & \eta_{n+1}=\zeta_{n}^{2}, \\
\zeta_{n+1}=\zeta_{n} \eta_{n}, & \eta_{n+1}=\eta_{n} \zeta_{n}, \\
\zeta_{n+1}=\eta_{n}^{2}, & \eta_{n+1}=\eta_{n}^{2}, \\
\zeta_{n+1}=\eta_{n}^{2}, & \eta_{n+1}=\eta_{n} \zeta_{n}, \\
\zeta_{n+1}=\eta_{n}^{2}, & \eta_{n+1}=\eta_{n}^{2}
\end{array}
$$

for $n \in \mathbb{N}_{0}$.

Now note that if we show the solvability of systems (18)-(26), this together with the two relations in (17) will show the solvability of systems (8)-(16). Because of this, our main task is to show that there are closed-form formulas for solutions to systems (18)-(26).

\section{Main results}

This section considers the problem of solvability of systems (18)-(26). The systems are considered separately, one by one. It is shown that they all are really solvable by presenting some closed-form formulas for their general solutions. As a consequence, some closed-form formulas for general solutions to systems (8)-(16) are obtained. In this way it is shown that each of the systems of difference equations is practically solvable.

\subsection{System (18)}

From the first equation in the system of difference equations (18) we have

$$
\zeta_{n}=\zeta_{n-1}^{2}, \quad n \in \mathbb{N}
$$

from which by iteration and a simple inductive argument we obtain

$$
\zeta_{n}=\zeta_{0}^{2^{n}}, \quad n \in \mathbb{N}_{0}
$$


By using (28) in the second equation in (18) it follows that

$$
\eta_{n}=\zeta_{0}^{2^{n}}, \quad n \in \mathbb{N}
$$

Using relations (28) and (29) in (17), we see that the following theorem holds.

Theorem 1 Let $a \neq 0$. Then the following closed-form formulas

$$
\begin{aligned}
& x_{n}=\sqrt{a} \frac{\left(\frac{x_{0}+\sqrt{a}}{x_{0}-\sqrt{a}}\right)^{2^{n}}+1}{\left(\frac{x_{0}+\sqrt{a}}{x_{0}-\sqrt{a}}\right)^{2^{n}}-1}, \quad n \in \mathbb{N}_{0}, \\
& y_{n}=\sqrt{a} \frac{\left(\frac{x_{0}+\sqrt{a}}{x_{0}-\sqrt{a}}\right)^{2^{n}}+1}{\left(\frac{x_{0}+\sqrt{a}}{x_{0}-\sqrt{a}}\right)^{2^{n}}-1}, \quad n \in \mathbb{N},
\end{aligned}
$$

present a general solution to system (8).

\subsection{System (19)}

Bearing in mind that the first equation in (19) is the same as in (18), we have that formula (28) also holds in this case. Employing (28) in the second equation in (19), we have

$$
\eta_{n}=\eta_{n-1} \zeta_{n-1}=\eta_{n-1} \zeta_{0}^{2^{n-1}}, \quad n \in \mathbb{N}
$$

From (30) and by a simple inductive argument, we obtain

$$
\eta_{n}=\eta_{0} \prod_{j=1}^{n} \zeta_{0}^{2^{j-1}}=\eta_{0} \zeta_{0}^{\sum_{j=1}^{n} 2^{j-1}}
$$

from which together with the formula for the sum of a finite geometric progression it follows that

$$
\eta_{n}=\eta_{0} \zeta_{0}^{2^{n}-1}, \quad n \in \mathbb{N}_{0}
$$

Using relations (28) and (31) in (17), we see that the following theorem holds.

Theorem 2 Let $a \neq 0$. Then the following closed-form formulas

$$
\begin{aligned}
& x_{n}=\sqrt{a} \frac{\left(\frac{x_{0}+\sqrt{a}}{x_{0}-\sqrt{a}}\right)^{2^{n}}+1}{\left(\frac{x_{0}+\sqrt{a}}{x_{0}-\sqrt{a}}\right)^{2^{n}-1}}, \quad n \in \mathbb{N}_{0}, \\
& y_{n}=\sqrt{a} \frac{\left(\frac{y_{0}+\sqrt{a}}{y_{0}-\sqrt{a}}\right)\left(\frac{x_{0}+\sqrt{a}}{x_{0}-\sqrt{a}}\right) 2^{2^{n}-1}+1}{\left(\frac{y_{0}+\sqrt{a}}{y_{0}-\sqrt{a}}\right)\left(\frac{x_{0}+\sqrt{a}}{x_{0}-\sqrt{a}}\right)^{2^{n}-1}-1}, \quad n \in \mathbb{N}_{0},
\end{aligned}
$$

present a general solution to system (9). 


\subsection{System (20)}

Since the first equation in (20) is the same as in (18), formula (28) also holds in this case. On the other hand, the second equation in (20) is obtained from the first one by interchanging letters $\zeta$ and $\eta$, from which along with (28) it follows that

$$
\eta_{n}=\eta_{0}^{2^{n}}, \quad n \in \mathbb{N}_{0} .
$$

Using relations (28) and (32) in (17), we see that the following theorem holds.

Theorem 3 Let $a \neq 0$. Then the following closed-form formulas

$$
\begin{aligned}
& x_{n}=\sqrt{a} \frac{\left(\frac{x_{0}+\sqrt{a}}{x_{0}-\sqrt{a}}\right)^{2^{n}}+1}{\left(\frac{x_{0}+\sqrt{a}}{x_{0}-\sqrt{a}}\right)^{2^{n}}-1}, \quad n \in \mathbb{N}_{0}, \\
& y_{n}=\sqrt{a} \frac{\left(\frac{y_{0}+\sqrt{a}}{y_{0}-\sqrt{a}}\right)^{2^{n}}+1}{\left(\frac{y_{0}+\sqrt{a}}{y_{0}-\sqrt{a}}\right)^{2^{n}}-1}, \quad n \in \mathbb{N}_{0},
\end{aligned}
$$

present a general solution to system (10).

\subsection{System (21)}

From the equations in (21) we have

$$
\zeta_{n}=\zeta_{n-1} \eta_{n-1}=\zeta_{n-1} \zeta_{n-2}^{2}, \quad n \geq 2
$$

If we use the following notations:

$$
a_{1}:=1 \text { and } \quad b_{1}:=2 \text {, }
$$

then equation (33) can be written as follows:

$$
\zeta_{n}=\zeta_{n-1}^{a_{1}} \zeta_{n-2}^{b_{1}}, \quad n \geq 2
$$

Employing relation (33), where index $n$ is replaced by $n-1$ in (34), we have

$$
\begin{aligned}
\zeta_{n} & =\zeta_{n-1}^{a_{1}} \zeta_{n-2}^{b_{1}}=\left(\zeta_{n-2} \zeta_{n-3}^{2}\right)^{a_{1}} \zeta_{n-2}^{b_{1}}=\zeta_{n-2}^{a_{1}+b_{1}} \zeta_{n-3}^{2 a_{1}} \\
& =\zeta_{n-2}^{a_{2}} \zeta_{n-3}^{b_{2}},
\end{aligned}
$$

where $a_{2}$ and $b_{2}$ are clearly defined by

$$
a_{2}:=a_{1}+b_{1} \quad \text { and } \quad b_{2}:=2 a_{1} \text {. }
$$

Relations (35) and (36) suggest that the following ones hold:

$$
\zeta_{n}=\zeta_{n-k}^{a_{k}} \zeta_{n-k-1}^{b_{k}}
$$


and

$$
a_{k}=a_{k-1}+b_{k-1}, \quad b_{k}=2 a_{k-1}
$$

for $2 \leq k \leq n-1$.

Indeed, assume that (37) and (38) hold for $k$ such that $2 \leq k \leq n-1$. Then, by using relation (33) where index $n$ is replaced by $n-k$ in (37), we have

$$
\begin{aligned}
\zeta_{n} & =\zeta_{n-k}^{a_{k}} \zeta_{n-k-1}^{b_{k}} \\
& =\left(\zeta_{n-k-1} \zeta_{n-k-2}^{2}\right)^{a_{k}} \zeta_{n-k-1}^{b_{k}} \\
& =\zeta_{n-k-1}^{a_{k}+b_{k}} \zeta_{n-k-2}^{2 a_{k}} \\
& =\zeta_{n-k-1}^{a_{k+1}} \zeta_{n-k-2}^{b_{k+1}},
\end{aligned}
$$

where $a_{k+1}$ and $b_{k+1}$ are clearly defined by

$$
a_{k+1}:=a_{k}+b_{k} \quad \text { and } \quad b_{k+1}:=2 a_{k}
$$

for $2 \leq k \leq n-2$. This inductive step along with (35) and (36) shows that (37) and (38) hold, as claimed.

Let $k=n-1$. Then from (37) we have

$$
\zeta_{n}=\zeta_{1}^{a_{n-1}} \zeta_{0}^{b_{n-1}}=\left(\zeta_{0} \eta_{0}\right)^{a_{n-1}} \zeta_{0}^{b_{n-1}}=\zeta_{0}^{a_{n-1}+b_{n-1}} \eta_{0}^{a_{n-1}}
$$

From this and since $a_{n-1}+b_{n-1}=a_{n}$, we have

$$
\zeta_{n}=\zeta_{0}^{a_{n}} \eta_{0}^{a_{n-1}}
$$

Now note that from (38) we have

$$
a_{n}=a_{n-1}+2 a_{n-2}, \quad n \geq 3 .
$$

Moreover, relation (40) can be used to calculate $a_{n}$ also for $n \leq 0$ by using the following obvious consequence of it:

$$
a_{n-2}=\frac{a_{n}-a_{n-1}}{2}
$$

Since $a_{1}=1$ and $a_{2}=3$, from (41) it follows that

$$
a_{0}=1 \text { and } a_{-1}=0
$$

By using (42) it is easy to see that formula (39) holds also for $n=0$.

The characteristic polynomial associated with equation (40) is $P_{2}(s)=s^{2}-s-2$, and clearly their roots are

$$
s_{1}=2 \text { and } s_{2}=-1 \text {. }
$$


Hence, by using the de Moivre formula (3), with $s_{1}$ and $s_{2}$ given in (43), and $c_{1}=2$ and $c_{2}=1$, we obtain

$$
a_{n}=\frac{2^{n+1}-(-1)^{n+1}}{3}, \quad n \in \mathbb{Z} .
$$

Using (44) in (39), we get

$$
\zeta_{n}=\zeta_{0}^{\frac{2^{n+1}-(-1)^{n+1}}{3}} \eta_{0}^{\frac{2^{n}-(-1)^{n}}{3}}, \quad n \in \mathbb{N}_{0}
$$

By using (45) in the second equation in (21), we obtain

$$
\eta_{n}=\zeta_{0}^{\frac{2^{n+1}-2(-1)^{n}}{3}} \eta_{0}^{\frac{2^{n}-2(-1)^{n-1}}{3}}, \quad n \in \mathbb{N}_{0}
$$

Using relations (45) and (46) in (17), we see that the following theorem holds.

Theorem 4 Let $a \neq 0$. Then the following closed-form formulas

$$
\begin{gathered}
x_{n}=\sqrt{a} \frac{\left(\frac{x_{0}+\sqrt{a}}{x_{0}-\sqrt{a}}\right)^{\frac{2^{n+1}-(-1)^{n+1}}{3}}\left(\frac{y_{0}+\sqrt{a}}{y_{0}-\sqrt{a}}\right)^{\frac{2^{n}-(-1)^{n}}{3}}+1}{\left(\frac{x_{0}+\sqrt{a}}{x_{0}-\sqrt{a}}\right)^{\frac{2^{n+1}-(-1)^{n+1}}{3}}\left(\frac{y_{0}+\sqrt{a}}{y_{0}-\sqrt{a}}\right)^{\frac{2^{n}-(-1)^{n}}{3}}-1}, \quad n \in \mathbb{N}_{0}, \\
y_{n}=\sqrt{a} \frac{\left(\frac{x_{0}+\sqrt{a}}{x_{0}-\sqrt{a}}\right)^{\frac{2^{n+1}-2(-1)^{n}}{3}}\left(\frac{y_{0}+\sqrt{a}}{y_{0}-\sqrt{a}}\right)^{\frac{2^{n}-2(-1)^{n-1}}{3}}+1}{\left(\frac{x_{0}+\sqrt{a}}{x_{0}-\sqrt{a}}\right)^{\frac{2^{n+1}-2(-1)^{n}}{3}}\left(\frac{y_{0}+\sqrt{a}}{y_{0}-\sqrt{a}}\right)^{\frac{2^{n}-2(-1)^{n-1}}{3}}-1}, \quad n \in \mathbb{N}_{0},
\end{gathered}
$$

present a general solution to system (11).

\subsection{System (22)}

First note that we have

$$
\zeta_{n}=\eta_{n}, \quad n \in \mathbb{N}
$$

Using (47) in the first equation in (22), we have that (27) holds, but this time for $n \geq 2$, from which it follows that

$$
\zeta_{n}=\zeta_{1}^{2^{n-1}}, \quad n \in \mathbb{N}
$$

and consequently,

$$
\zeta_{n}=\zeta_{0}^{2^{n-1}} \eta_{0}^{2^{n-1}}, \quad n \in \mathbb{N}
$$

From (47) and (48) we have

$$
\eta_{n}=\zeta_{0}^{2^{n-1}} \eta_{0}^{2^{n-1}}, \quad n \in \mathbb{N}
$$

Using relations (48) and (49) in (17), we see that the following theorem holds. 
Theorem 5 Let $a \neq 0$. Then the following closed-form formulas

$$
\begin{aligned}
& x_{n}=\sqrt{a} \frac{\left(\frac{x_{0}+\sqrt{a}}{x_{0}-\sqrt{a}}\right)^{2^{n-1}}\left(\frac{y_{0}+\sqrt{a}}{y_{0}-\sqrt{a}}\right)^{2^{n-1}}+1}{\left(\frac{x_{0}+\sqrt{a}}{x_{0}-\sqrt{a}}\right)^{2^{n-1}\left(\frac{y_{0}+\sqrt{a}}{y_{0}-\sqrt{a}}\right)^{2^{n-1}-1}}, \quad n \in \mathbb{N},} \\
& y_{n}=\sqrt{a} \frac{\left(\frac{x_{0}+\sqrt{a}}{x_{0}-\sqrt{a}}\right)^{2^{n-1}}\left(\frac{y_{0}+\sqrt{a}}{y_{0}-\sqrt{a}}\right)^{2^{n-1}+1}}{\left(\frac{x_{0}+\sqrt{a}}{x_{0}-\sqrt{a}}\right)^{2^{n-1}}\left(\frac{y_{0}+\sqrt{a}}{y_{0}-\sqrt{a}}\right)^{2^{n-1}-1}}, \quad n \in \mathbb{N},
\end{aligned}
$$

present a general solution to system (12).

\subsection{System (23)}

This system is obtained from the system in (19) by interchanging letters $\zeta$ and $\eta$. Hence, we have that the following formulas hold:

$$
\zeta_{n}=\zeta_{0} \eta_{0}^{2^{n}-1}, \quad n \in \mathbb{N}_{0}
$$

and

$$
\eta_{n}=\eta_{0}^{2^{n}}, \quad n \in \mathbb{N}_{0}
$$

Using relations (50) and (51) in (17), we see that the following theorem holds.

Theorem 6 Let $a \neq 0$. Then the following closed-form formulas

$$
\begin{aligned}
& x_{n}=\sqrt{a} \frac{\left(\frac{x_{0}+\sqrt{a}}{x_{0}-\sqrt{a}}\right)\left(\frac{y_{0}+\sqrt{a}}{y_{0}-\sqrt{a}}\right)^{2^{n}-1}+1}{\left(\frac{x_{0}+\sqrt{a}}{x_{0}-\sqrt{a}}\right)\left(\frac{y_{0}+\sqrt{a}}{y_{0}-\sqrt{a}}\right)^{2^{n}-1}-1}, \quad n \in \mathbb{N}_{0}, \\
& y_{n}=\sqrt{a} \frac{\left(\frac{y_{0}+\sqrt{a}}{y_{0}-\sqrt{a}}\right)^{2^{n}+1}}{\left(\frac{y_{0}+\sqrt{a}}{y_{0}-\sqrt{a}}\right)^{2^{n}}-1}, \quad n \in \mathbb{N}_{0},
\end{aligned}
$$

present a general solution to system (13).

\subsection{System (24)}

By using the second equation in (24) in the first one, we obtain

$$
\zeta_{n}=\eta_{n-1}^{2}=\zeta_{n-2}^{4}, \quad n \geq 2
$$

From (52) we have

$$
\zeta_{2 n}=\zeta_{2(n-1)}^{4}, \quad n \in \mathbb{N}
$$

from which by iteration and a simple inductive argument we obtain

$$
\zeta_{2 n}=\zeta_{0}^{4^{n}}, \quad n \in \mathbb{N}_{0}
$$


and

$$
\zeta_{2 n+1}=\zeta_{2(n-1)+1}^{4}, \quad n \in \mathbb{N},
$$

from which by iteration and a simple inductive argument we obtain

$$
\zeta_{2 n+1}=\zeta_{1}^{4^{n}}=\eta_{0}^{2^{2 n+1}}, \quad n \in \mathbb{N}_{0}
$$

Since this system is symmetric, we have

$$
\eta_{2 n}=\eta_{0}^{4^{n}}, \quad n \in \mathbb{N}_{0}
$$

and

$$
\eta_{2 n+1}=\zeta_{0}^{2 n+1}, \quad n \in \mathbb{N}_{0}
$$

Using relations (53)-(56) in (17), we see that the following theorem holds.

Theorem 7 Let $a \neq 0$. Then the following closed-form formulas

$$
\begin{aligned}
& x_{2 n}=\sqrt{a} \frac{\left(\frac{x_{0}+\sqrt{a}}{x_{0}-\sqrt{a}}\right)^{4^{n}}+1}{\left(\frac{x_{0}+\sqrt{a}}{x_{0}-\sqrt{a}}\right)^{4^{n}}-1}, \quad n \in \mathbb{N}_{0}, \\
& x_{2 n+1}=\sqrt{a} \frac{\left(\frac{y_{0}+\sqrt{a}}{y_{0}-\sqrt{a}}\right)^{2^{2 n+1}+1}}{\left(\frac{y_{0}+\sqrt{a}}{y_{0}-\sqrt{a}}\right)^{2^{2 n+1}-1}}, \quad n \in \mathbb{N}_{0}, \\
& y_{2 n}=\sqrt{a} \frac{\left(\frac{y_{0}+\sqrt{a}}{y_{0}-\sqrt{a}}\right)^{4^{n}}+1}{\left(\frac{y_{0}+\sqrt{a}}{y_{0}-\sqrt{a}}\right)^{4^{n}}-1}, \quad n \in \mathbb{N}_{0}, \\
& y_{2 n+1}=\sqrt{a} \frac{\left(\frac{x_{0}+\sqrt{a}}{x_{0}-\sqrt{a}}\right)^{2^{2 n+1}}+1}{\left(\frac{x_{0}+\sqrt{a}}{x_{0}-\sqrt{a}}\right)^{2^{2 n+1}}-1}, \quad n \in \mathbb{N}_{0},
\end{aligned}
$$

present a general solution to system (14).

\subsection{System (25)}

This system is obtained from the system in (21) by interchanging letters $\zeta$ and $\eta$. Hence, we have that the following formulas hold:

$$
\zeta_{n}=\eta_{0}^{\frac{2^{n+1}-2(-1)^{n}}{3}} \zeta_{0}^{\frac{2^{n}-2(-1)^{n-1}}{3}}
$$

and

$$
\eta_{n}=\eta_{0}^{\frac{2^{n+1}-(-1)^{n+1}}{3}} \zeta_{0}^{\frac{2^{n}-(-1)^{n}}{3}}
$$

Using relations (57) and (58) in (17), we see that the following theorem holds. 
Theorem 8 Let $a \neq 0$. Then the following closed-form formulas

$$
\begin{aligned}
& x_{n}=\sqrt{a} \frac{\left(\frac{y_{0}+\sqrt{a}}{y_{0}-\sqrt{a}}\right){\frac{2^{n+1}-2(-1)^{n}}{3}}^{\left.\frac{y_{0}+\sqrt{a}}{x_{0}-\sqrt{a}}\right)^{\frac{2^{n}-2(-1)^{n-1}}{3}}+1}}{\left(\frac{y_{0}+\sqrt{a}}{y_{0}-\sqrt{a}}\right) \frac{2^{n+1}-2(-1)^{n}}{3}\left(\frac{x_{0}+\sqrt{a}}{x_{0}-\sqrt{a}}\right)^{\frac{2^{n}-2(-1)^{n-1}}{3}}-1}, \quad n \in \mathbb{N}_{0}, \\
& y_{n}=\sqrt{a} \frac{\left(\frac{y_{0}+\sqrt{a}}{y_{0}-\sqrt{a}}\right)^{\frac{2^{n+1}-(-1)^{n+1}}{3}}\left(\frac{x_{0}+\sqrt{a}}{x_{0}-\sqrt{a}}\right)^{\frac{2^{n}-(-1)^{n}}{3}}+1}{\left(\frac{y_{0}+\sqrt{a}}{y_{0}-\sqrt{a}}\right)^{\frac{2^{n+1}-(-1)^{n+1}}{3}}\left(\frac{x_{0}+\sqrt{a}}{x_{0}-\sqrt{a}}\right)^{\frac{2^{n}-(-1)^{n}}{3}}-1}, \quad n \in \mathbb{N}_{0},
\end{aligned}
$$

present a general solution to system (15).

\subsection{System (26)}

This system is obtained from the system in (18) by interchanging letters $\zeta$ and $\eta$. Hence, we have that the following formulas hold:

$$
\zeta_{n}=\eta_{0}^{2^{n}}, \quad n \in \mathbb{N}, \quad \text { and } \quad \eta_{n}=\eta_{0}^{2^{n}}, \quad n \in \mathbb{N}_{0}
$$

Using (59) in (17), we see that the following theorem holds.

Theorem 9 Let $a \neq 0$. Then the following closed-form formulas

$$
\begin{aligned}
& x_{n}=\sqrt{a} \frac{\left(\frac{y_{0}+\sqrt{a}}{y_{0}-\sqrt{a}}\right)^{2^{n}}+1}{\left(\frac{y_{0}+\sqrt{a}}{y_{0}-\sqrt{a}}\right)^{2^{n}}-1}, \quad n \in \mathbb{N}, \\
& y_{n}=\sqrt{a} \frac{\left(\frac{y_{0}+\sqrt{a}}{y_{0}-\sqrt{a}}\right)^{2^{n}}+1}{\left(\frac{y_{0}+\sqrt{a}}{y_{0}-\sqrt{a}}\right)^{2^{n}}-1}, \quad n \in \mathbb{N}_{0},
\end{aligned}
$$

present a general solution to system (16).

\section{Acknowledgements}

Not applicable.

Funding

Not applicable.

Availability of data and materials

Not applicable.

\section{Competing interests}

The author declares that he has no competing interests.

\section{Authors' contributions}

The author has contributed solely to the writing of this paper. He read and approved the manuscript.

\section{Publisher's Note}

Springer Nature remains neutral with regard to jurisdictional claims in published maps and institutional affiliations.

Received: 1 October 2020 Accepted: 30 November 2020 Published online: 09 December 2020

\section{References}

1. de Moivre, A.: Miscellanea Analytica de Seriebus et Quadraturis. J. Tonson \& J. Watts, Londini (1730) (in Latin)

2. Euler, L.: Introductio in Analysin Infinitorum, Tomus Primus, Lausannae (1748) (in Latin)

3. Fort, T.: Finite Differences and Difference Equations in the Real Domain. Oxford University Press, London (1948)

4. Jordan, C.: Calculus of Finite Differences. Chelsea, New York (1956) 
5. Markoff, A.A.: Differenzenrechnung. Teubner, Leipzig (1896) (in German)

6. Milne-Thomson, L.M.: The Calculus of Finite Differences. Macmillan \& Co., London (1933)

7. Mitrinović, D.S., Kečkić, J.D.: Metodi Izračunavanja Konačnih Zbirova/Methods for Calculating Finite Sums. Naučna Knjiga, Beograd (1984) (in Serbian)

8. Nörlund, N.E.: Vorlesungen über Differenzenrechnung. Springer, Berlin (1924) (in German)

9. Berg, L., Stević, S.: On some systems of difference equations. Appl. Math. Comput. 218, 1713-1718 (2011)

10. Papaschinopoulos, G., Stefanidou, G.: Asymptotic behavior of the solutions of a class of rational difference equations. Int. J. Difference Equ. 5(2), 233-249 (2010)

11. Stević, S.: On some solvable systems of difference equations. Appl. Math. Comput. 218, 5010-5018 (2012)

12. Stević, S.: Sixteen practically solvable systems of difference equations. Adv. Differ. Equ. 2019, Article ID 467 (2019)

13. Stević, S.: Solvability of a general class of two-dimensional hyperbolic-cotangent-type systems of difference equations. Adv. Differ. Equ. 2019, Article ID 294 (2019)

14. Stević, S., Diblik, J., Iričanin, B., Šmarda, Z.: On some solvable difference equations and systems of difference equations. Abstr. Appl. Anal. 2012, Article ID 541761 (2012)

15. Stević, S., Iričanin, B., Kosmala, W.: More on a hyperbolic-cotangent class of difference equations. Math. Methods Appl. Sci. 42, 2974-2992 (2019)

16. Stević, S., Iričanin, B., Kosmala, W., Šmarda, Z.: Note on the bilinear difference equation with a delay. Math. Methods Appl. Sci. 41, 9349-9360 (2018)

17. Stević, S., Iričanin, B., Kosmala, W., Šmarda, Z.: Representation of solutions of a solvable nonlinear difference equation of second order. Electron. J. Qual. Theory Differ. Equ. 2018, Article ID 95 (2018)

18. Stević, S., Tollu, D.T: Solvability and semi-cycle analysis of a class of nonlinear systems of difference equations. Math. Methods Appl. Sci. 42, 3579-3615 (2019)

19. Stević, S., Tollu, D.T.: Solvability of eight classes of nonlinear systems of difference equations. Math. Methods Appl. Sci. 42, 4065-4112 (2019)

20. Papaschinopoulos, G., Schinas, C.J.: On the behavior of the solutions of a system of two nonlinear difference equations. Commun. Appl. Nonlinear Anal. 5(2), 47-59 (1998)

21. Papaschinopoulos, G., Schinas, C.J.: Invariants for systems of two nonlinear difference equations. Differ. Equ. Dyn. Syst. 7, 181-196 (1999)

22. Papaschinopoulos, G., Schinas, C.J.: Invariants and oscillation for systems of two nonlinear difference equations. Nonlinear Anal., Theory Methods Appl. 46, 967-978 (2001)

23. Papaschinopoulos, G., Schinas, C.J., Stefanidou, G.: On a k-order system of Lyness-type difference equations. Adv. Differ. Equ. 2007, Article ID 31272 (2007)

24. Schinas, C.: Invariants for difference equations and systems of difference equations of rational form. J. Math. Anal. Appl. 216, 164-179 (1997)

25. Schinas, C.: Invariants for some difference equations. J. Math. Anal. Appl. 212, 281-291 (1997)

26. Stević, S.: Solvability of some classes of nonlinear first-order difference equations by invariants and generalized invariants. Electron. J. Qual. Theory Differ. Equ. 2019, Article ID 36 (2019)

27. Stević, S.: Solving a class of non-autonomous difference equations by generalized invariants. Math. Methods Appl. Sci. 42, 6315-6338 (2019)

28. Berezansky, L., Braverman, E.: On impulsive Beverton-Holt difference equations and their applications. J. Differ. Equ. Appl. 10(9), 851-868 (2004)

29. Levy, H., Lessman, F.: Finite Difference Equations. Dover, New York (1992)

30. Proskuryakov, I.V.: Problems in Linear Algebra. Nauka, Moscow (1984) (in Russian)

31. Riordan, J.: Combinatorial Identities. Wiley, New York (1968)

32. Vorobiev, N.N.: Fibonacci Numbers. Birkhäuser, Basel (2002)

33. Laplace, P.S.: Recherches sur l'intégration des équations différentielles aux différences finies et sur leur usage dans la théorie des hasards. Mémoires de l' Académie Royale des Sciences de Paris 1773, t. VII, (1776) (Laplace OEuvres, VIII, 69-197, 1891). (in French)

34. Papaschinopoulos, G., Schinas, C.J.: On a system of two nonlinear difference equations. J. Math. Anal. Appl. 219(2) 415-426 (1998)

35. Papaschinopoulos, G., Schinas, C.J.: Oscillation and asymptotic stability of two systems of difference equations of rational form. J. Differ. Equ. Appl. 7, 601-617 (2001)

36. Papaschinopoulos, G., Schinas, C.J.: On the system of two difference equations $x_{n+1}=\sum_{i=0}^{k} A_{i} / y_{n-i}^{p_{i}} y_{n+1}=\sum_{i=0}^{k} B_{i} / x_{n-i}^{q_{i}}$ J. Math. Anal. Appl. 273(2), 294-309 (2002)

37. Papaschinopoulos, G., Schinas, C.J.: On the dynamics of two exponential type systems of difference equations. Comput. Math. Appl. 64(7), 2326-2334 (2012)

38. Stević, S.: New class of practically solvable systems of difference equations of hyperbolic-cotangent-type. Electron. J. Qual. Theory Differ. Equ. 2020 (2020) (to appear)

39. Stević, S.: Solvable product-type system of difference equations whose associated polynomial is of the fourth order. Electron. J. Qual. Theory Differ. Equ. 2017, Article ID 13 (2017) 